\title{
Physiological Properties, Time of Development, and Central Projection Are Correlated in the Wing Mechanoreceptors of Drosophila
}

\author{
Michael H. Dickinson and John Palka \\ Department of Zoology, University of Washington, Seattle, Washington 98195
}

\begin{abstract}
The wing of Drosophila contains 8 sensory structures (campaniform sensilla), which lie in specific locations and possess identical surface morphology. The axons of the campaniform neurons follow either a medial or a lateral tract within the CNS. Previous studies (Palka et al., 1986) indicate that choice of central pathway correlates with the time of birth and differentiation of the neurons rather than with their topographic distribution on the wing. On the basis of the response properties revealed by mechanical and electrical stimulation, these sensory cells also fall into 2 physiological categories, rapidly and slowly adapting, that correlate exactly with central projection and birthdate. Thus, within this discrete population of sensory neurons there exists a precise 3-way correlation between physiology, central projection, and time of development.
\end{abstract}

The sensory systems of insects, like those of vertebrates, are often characterized by the presence of spatially ordered central projections - topographic maps of the sensory surface projected upon CNS (Strausfeld, 1976; Murphey, 1981, 1985). However, topography is not the only ordering principle in these systems. For example, we have previously described in the wings of Drosophila a set of 8 structurally indistinguishable campaniform sensilla (sensory structures that detect deformation of the cuticle) whose axons form 2 distinct tracts and branching patterns within the CNS (Palka et al., 1986). The neurons whose axons travel and branch together are never closest neighbors on the wing surface, so that a conventional topographic representation is not formed. However, the central projections of these sensory neurons are segregated according to the time of birth and differentiation of their cell bodies in the wing, which occur in 2 discrete waves. The axons of the 4 early-developing neurons follow the medial tract within the CNS, and those of the 4 latedeveloping neurons follow the lateral tract. We report here that this correlation of central pathway with time of development also reflects physiological function: the 4 early-developing neurons adapt rapidly in response to mechanical or electrical stimulation, whereas the later-developing ones adapt slowly. Thus,

\footnotetext{
Received Mar. 18, 1987; revised June 8, 1987; accepted June 11, 1987.

We thank Dr. M. A. Murray for use of photomicrographs, and J. Stewart for help with construction of wing maps. Drs. T. Daniel and W. Moody provided helpful criticism of this manuscript. This research was supported by NIH Jacob Javits Award NS 07778 and NSF Research Grant BNS 8507460 (to J.P.), and NSF Graduate Fellowship (to M.H.D.).

Correspondence should be addressed to Michael $H$. Dickinson at the above address.

Copyright (c) 1987 Society for Neuroscience $0270-6474 / 87 / 124201-08 \$ 02.00 / 0$
}

there is a 3-way correlation between physiology, central projections and time of development in this system. Preliminary accounts of some of this work have appearcd clscwhere (Dickinson and Palka, 1985, 1986).

\section{Materials and Methods}

Animals. The Oregon-R strain of wild-type $D$. melanogaster was used in this study. Other species of Drosophila were originally obtained from the National Drosophila Species Resource Center in Bowling Green, Ohio. All drosophilids were raised on standard $D$. melanogaster medium. Colonies of Calliphora sp. were started with larvae obtained from College Biological Supply Co. Pupae of Musca domestica and Sarcophaga bullata were obtained from Carolina Biological Supply Co. The larvae of these larger flies were raised for the first day on chicken liver and subsequently on a mixture of corn meal, yeast, agar, and water. Adults were fed sugar and dry milk mixed with cholesterol.

Electrophysiology. The technique used to make extracellular recordings from campaniform sensory neurons is shown in Figure 1. The wing was removed from the thorax, leaving a small section of the pleura and notum attached. The wing tip was cut off, providing electrical access to the longitudinal veins, which contain the axons of the sensory neurons. In recordings using electrical stimulation a small notch was also cut in the margin of the wing, providing electrical contact for a ground electrode. The wing was positioned over 2 parallel insect pins, which were glued to a Plexiglass stage and thinly coated with petroleum jelly. Electrical contact with the wing was made via 3 pools of insect saline (Levine, 1966) held in wells milled into the stage, located such that fluid contact would be made with the proximal part of the wing, the third vein at the distal cut, and the small notch in the margin when a current return was nccessary. An AC-coupled, high input impedance amplifier was used to record action potentials in the wing neurons, with active electrodes placed in the saline pools at the proximal and distal ends of the wing. A high input impedance was necessary because the resistance between the recording electrodes was typically 2-5 M 2 . In most experiments, permanent records were obtained by photography. Some experiments were recorded using an FM magnetic tape recorder and replayed for subsequent analysis. An Ortec 4620/4621 event counter coupled with a window discriminator was used to produce frequency histograms of action potentials.

Stimulation. Mechanical stimulation was carried out using a probe that produced precisely controlled motion with simultaneous measurement of stimulus force. The force transducer consisted of 2 semiconductor strain gauges, mounted on opposite sides of a small rectangular piece of 0.003 inch stainless steel shim and arranged as complementary arms in a bridge circuit. This transducer was glued on one end to an electrolytically sharpened tungsten needle and on the other end to a piezoelectric crystal mounted in a micromanipulator. Voltage applied across the crystal resulted in movement of the tungsten probe, which could be calibrated as stress measured by the serially arranged transducer. The probe was positioned under compound microscope optics, using a $40 \times$ long-working-distance objective and $15 \times$ eyepieces. The wings of Calliphora, Musca, and Sarcophaga are large and relatively stiff, which facilitates discrete stimulation of a single campaniform neuron by the application of force directly to the dome of the sensillum (Chapman et al., 1973). However, campaniform neurons are extremely sensitive to displacement of the dome [the threshold sensitivity of cock- 


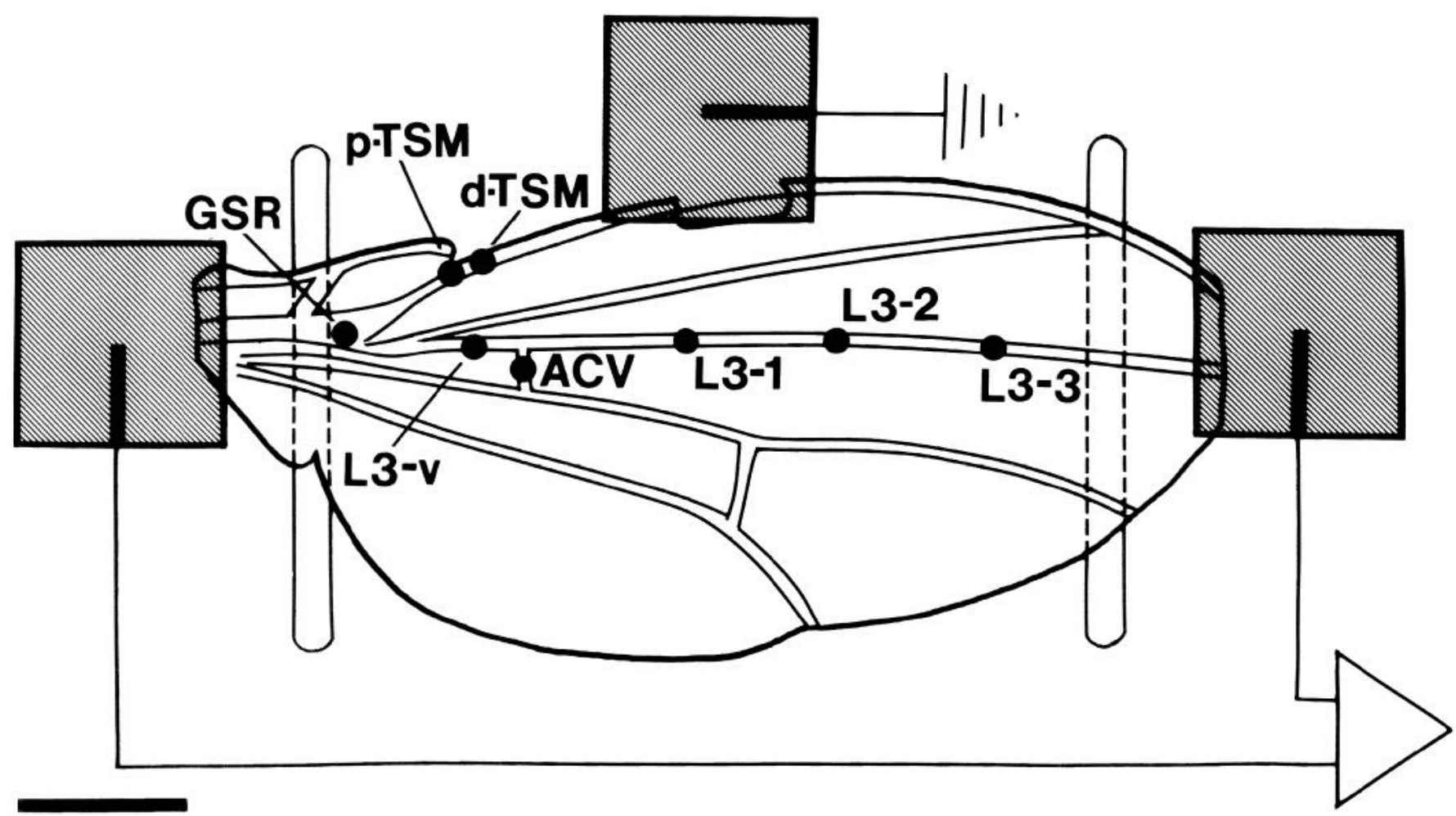

Figure 1. Arrangement for extracellular recording of spikes from campaniform sensory neurons. The wing is positioned over 2 insect pins coated with petroleum jelly that hold it in place. Electrical contact is made with the hemolymph of the veins via cuts in the cuticle and 3 pools of insect saline (cross-hatched). The 8 structurally identical sensilla are shown in positions characteristic for Drosophila; for other genera, see Figure 7. Abbreviations: GSR, giant sensillum of the radius (the thick vein at the base of the wing); $p$-TSM and $d$-TSM, proximal and distal twin sensilla of the margin (the large vein at the anterior edge of the wing); $L 3-v$, ventral sensillum of L3 (the third longitudinal vein); $A C V$, sensillum of the anterior cross vein (the short vein bridging L3 and L4); L3-I, L3-2, L3-3, first, second, and third sensilla of L3. All of the campaniform sensilla except L3-v are on the dorsal surface of the wing. Calibration bar, $400 \mu \mathrm{m}$.
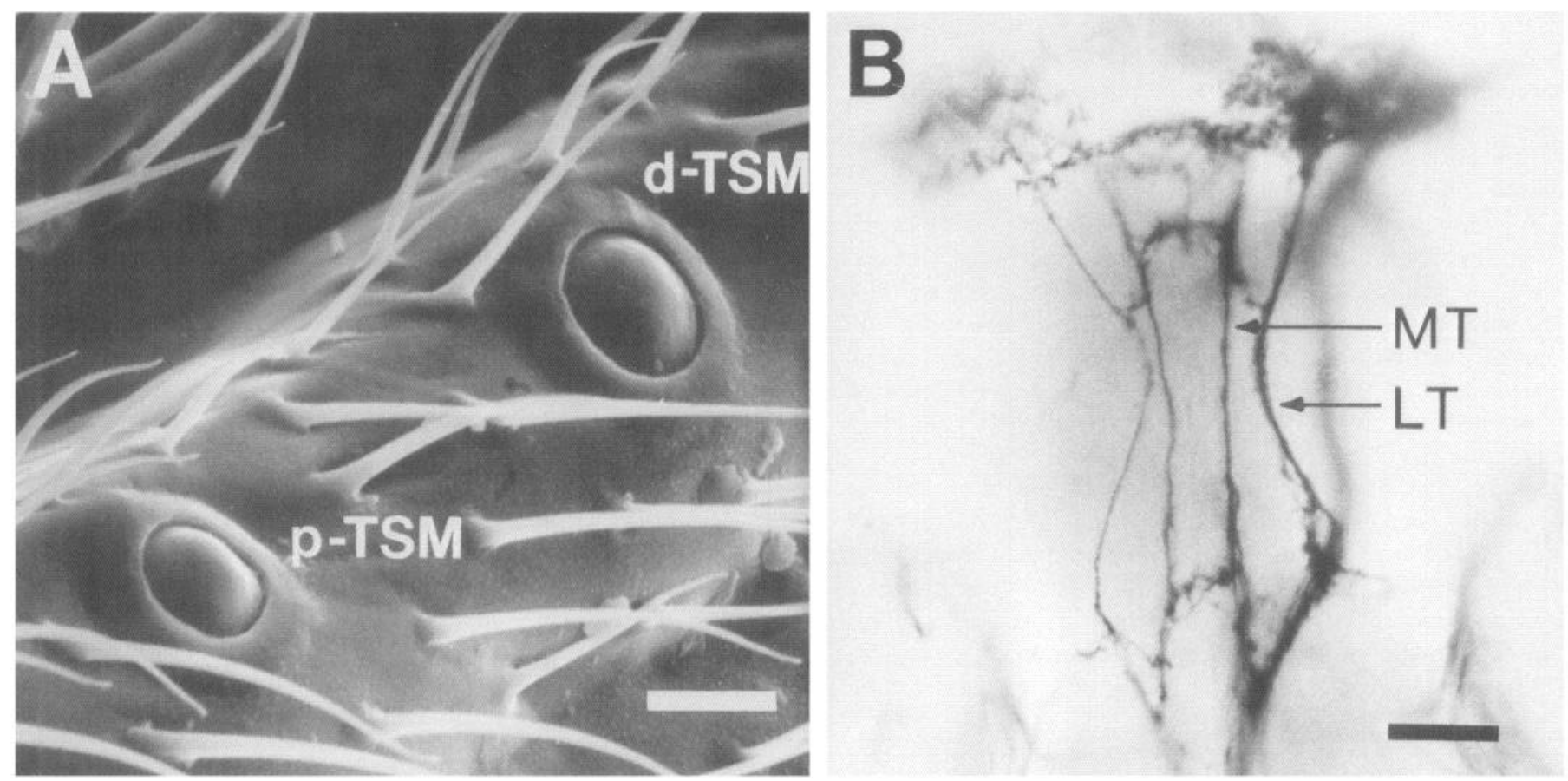

Figure 2. External morphology and central projections of campaniform sensilla in D. melanogaster. A, Scanning electron micrograph of the marginal vein showing the surface morphology of the twin sensilla. d-TSM belongs to the early-developing group, p-TSM to the late-developing group. Although the sensilla are similar in external appearance, their neurons follow different pathways within the CNS and have different physiological properties. Calibration bar, $5 \mu \mathrm{m}$. B, Central projections from campaniform sensilla (cobalt backfills intensified by modified Timm's method; Palka et al., 1986). The axons of campaniform neurons that differentiate early (d-TSM, GSR, ACV, L3-2) follow the medial tract (MT), while those of cells that differentiate later (p-TSM, L3-v, L3-1, L3-3) follow the lateral tract (LT). Calibration bar, $100 \mu \mathrm{m}$. 

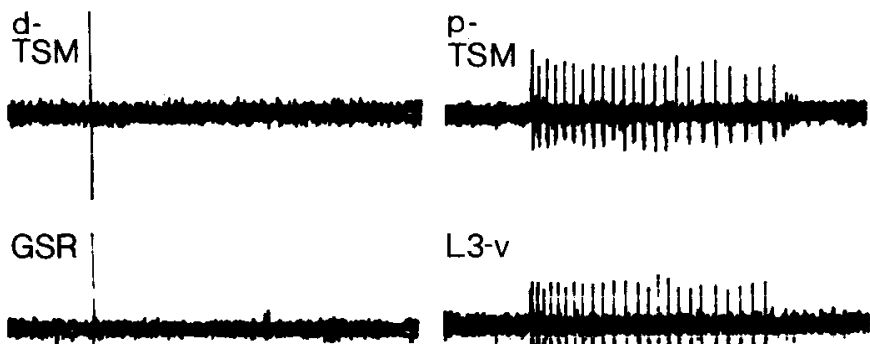

L3-v
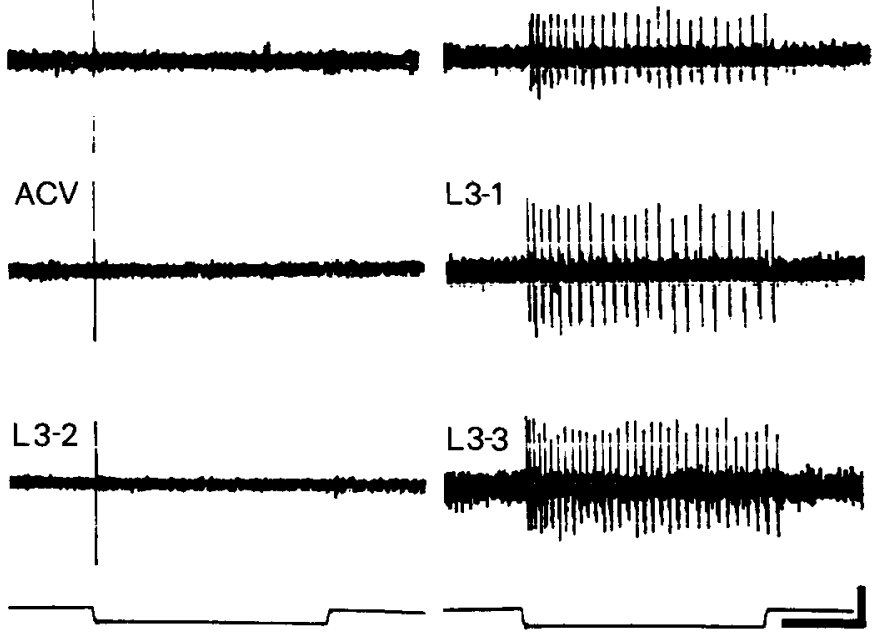

Figure 3. Extracellular recordings from the wing of $D$. virilis showing the responses of individual campaniform sensilla to deformational stimuli. The stimulus probe delivered a $50-75 \mu \mathrm{N}$ force to the vein cuticle immediately adjacent to the sensillum in each case. Only one characteristic force record is included for each set of recordings. The 4 earlydeveloping cells respond with a single impulse at the onset of the trapezoidal deflection. The 4 late-developing cells continue to fire for the duration of the deformation with little decrease in firing frequency. Calibration bar, $100 \mu \mathrm{V}$ for left column, $50 \mu \mathrm{V}$ for right column; 200 msec for all traces.

roach tibial campaniform sensilla has been estimated at $1 \mathrm{~nm}$ (Chapman and Duckrow, 1975)], and neurons often responded to background vibrations of the building when the stimulus probe was placed directly on the campaniform dome. For this reason the force probe was commonly placed on the vein cuticle immediately adjacent to a sensillum. This is also a behaviorally more relevant stimulus, since these sensilla normally encode deformation of the cuticle rather than direct indentation of the dome. Local stimulation was more difficult on the smaller and more compliant wings of Drosophila spp., where even small stimuli often excited neighboring campaniform cells. However, unambiguous recording from single neurons could be made by cutting the wing into small sections and/or crushing particular sensilla, leaving the single sensillum of interest intact.

Electrical stimulation of campaniform neurons was accomplished across the cuticle with inward positive current supplied by a Getting model 5 microelectrode amplifier. Micropipettes were broken and filled with Aquasonic electric contact jelly (Parker Laboratories). Single campaniform sensilla were covered with a small pool of jelly extruded from the micropipette using a micrometer syringe. The current return path was supplied by a saline pool in contract with a notch in the margin of the wing as described above. The stimulating current was monitored by a virtual ground circuit in series with the return path.

\section{Results}

\section{Anatomical characteristics}

Figure 1 indicates the position of the 8 apparently identical campaniform sensilla of the wing blade of Drosophila. These data were obtained from $D$. melanogaster, but we have found that the pattern of sensilla placement is virtually identical in another 45 species within the genus, 7 species of 5 other genera within the family Drosophilidac, and the muscoid flies Calliphora sp., $M$. domestica, and $S$. bullata (see also Fig. 7 below).
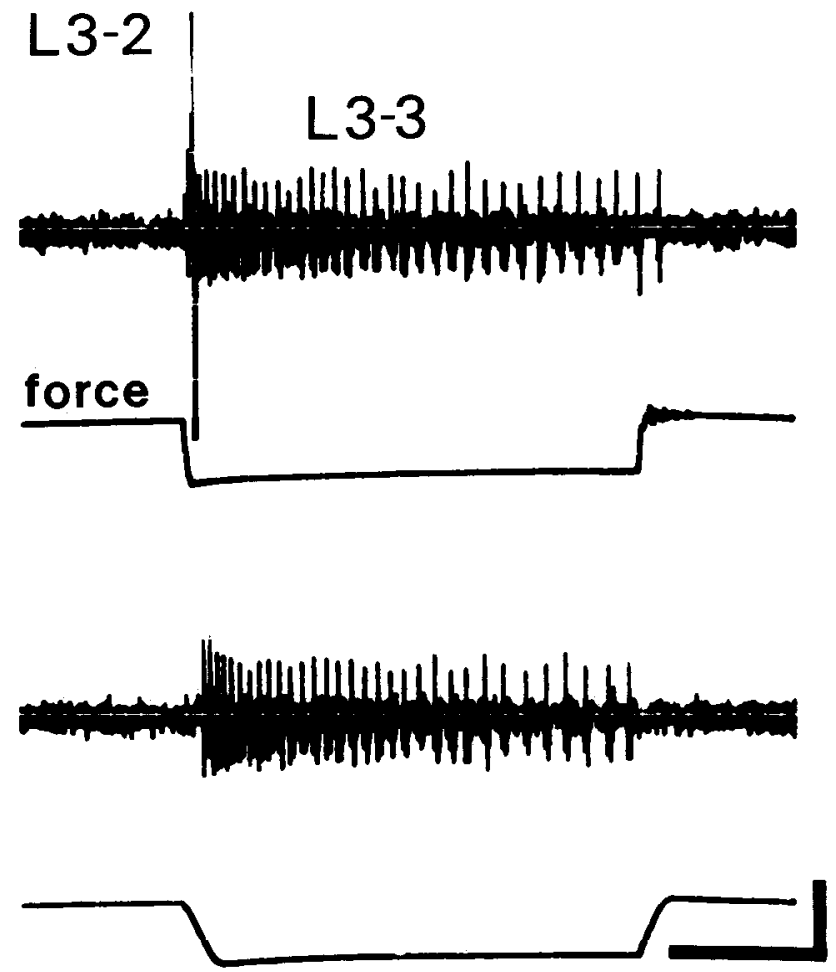

Figure 4. Extracellular recordings from the wing of $D$. virilis indicating the effects of stimulus slope on the response of rapidly and slowly adapting neurons. The force probe was placed on the third vein at the midpoint between L3-2 and L3-3, and a large force (200 $\mu \mathrm{N}$ peak) was applied. In the upper trace the force record indicates an initial stimulus slope of $20 \mu \mathrm{N} / \mathrm{msec}$. This deflection velocity was sufficient to elicit a response from both neurons. At a decreased deformation slope of $4 \mu \mathrm{N} /$ msec (lower trace), the L3-2 neuron failed to respond. In both instances, the slowly adapting L3-3 neuron fired impulses for the duration of the stimulus. The wing slowly springs back from the stimulus stress, as indicated by the small relaxations in the force records. Calibration bar, $50 \mu \mathrm{V}, 200 \mathrm{msec}$.

We used $D$. virilis in the majority of physiological experiments described here because their wings are slightly longer and stiffer, and the extracellularly recorded impulses are larger, than those of $D$. melanogaster. However, all the experiments were repeated on $D$. melanogaster, and no differences between the 2 species were observed.

The extreme similarity in external morphology of the earlyand late-developing sensilla is illustrated in Figure $2 A$, which shows a scanning electron micrograph of the twin sensilla of the margin (for their location on the wing, see Figs. 1 and 2). The distal sensillum (d-TSM) belongs to the early group, while the proximal one (p-TSM) belongs to the late group. They are indistinguishable except for the slightly greater diameter of the d-TSM. This minor difference is a regular feature of these 2 sensilla but does not represent a consistent difference between the early and late groups. The 2 tracts followed by the axons of the early- and late-developing neurons, the medial tract and lateral tract, respectively, are shown in a cobalt-filled preparation in Figure $2 B$.

\section{Mechanical stimulation}

Figure 3 shows recordings from the 8 campaniform neurons of $D$. virilis. The sensilla were individually stimulated by trapezoidal force pulses applied to the vein near the campaniform 

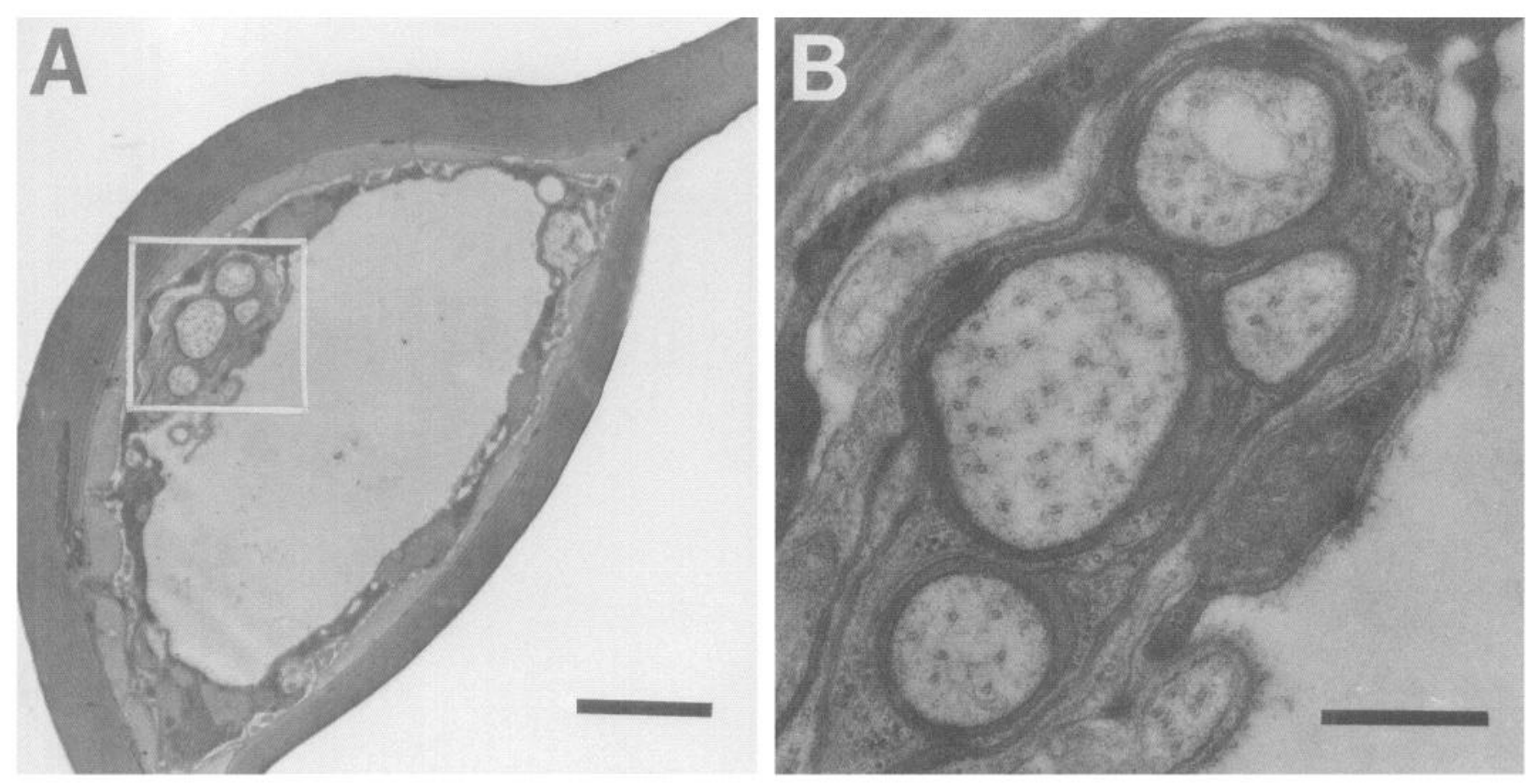

Figure 5. Size differences among the axons of campaniform neurons. A, Transmission electron micrograph showing a cross section through vein L3 just proximal to the anterior cross vein in D. melanogaster. A cluster of 4 axons is seen on the dorsal surface within the vein. Calibration bar, $2 \mu \mathrm{m}$. B , Enlargement of region indicated in $A$. Judging by the size of extracellular impulses, the 2 largest axons probably belong to the ACV and L3-2 neurons, and 2 smaller axons belong to the L3-1 and L3-3 cells. Calibration bar, $0.5 \mu \mathrm{m}$. Protocol for microscopy as described by Murray et al. (1984). Micrographs were kindly provided by Dr. Marjorie A. Murray.

dome. Four of the sensory neurons (d-TSM, GSR, ACV, and L3-2) adapted rapidly to the constant strain, responding with a single large action potential at the onset of deformation. Stronger deformations often resulted in a doublet of spikes at the onset of stimulation and a single impulse at the offset. The other 4 neurons (p-TSM, L3-v, L3-1, and L3-3) responded much more tonically, continuing to fire action potentials for the duration of the stimulus. The impulse activity of these neurons would continue during stimuli lasting $30 \mathrm{sec}$, with a very slow decline in firing rate after the first few seconds.

The rapidly and slowly adapting neurons also differ with respect to their sensitivity to the rate of deformation. As illustrated in Figure 4, trapezoidal deformations with steep initial slopes ( $>20 \mu \mathrm{N} / \mathrm{msec}$ ) elicited responses from both neurons, whereas slowly applied deformations $(<4 \mu \mathrm{N} / \mathrm{msec})$ excited the slowly adapting cells but usually failed to excite the rapidly adapting ones. The slowly adapting neurons appear best suited for encoding the magnitude of slow wing deformations, while the rapidly adapting cells respond best to fast transients and highfrequency oscillations. A detailed frequency analysis will be presented separately.

The impulses of the slowly adapting neurons were usually at least $50 \%$ smaller in amplitude than those of the rapidly adapting neurons, suggesting a smaller axonal diameter. This interpretation is consistent with electron microscopic cross sections made just proximal to the anterior cross vein. These reveal 2 size classes among the axons of the third vein (Fig. 5). The largest axon probably belongs to the ACV neuron, which appears to have the largest axon of all the campaniform neurons, judging by its appearance when single-cell fills were made by cobalt injection (Palka et al., 1986). By elimination, the second largest axon in the cross section is most likely to be the rapidly adapting L3-2. The 2 smaller axons would then belong to the L3-1 and L3-3 neurons, though it is not possible to distinguish between them.

The division of these 8 cells into 2 physiological classes correlates precisely with the dichotomy in birthdate and central projection identified in D. melanogaster (Murray et al., 1984; Palka et al., 1986). All 4 rapidly adapting neurons belong to the early developmental class of cells, which sends axons in the medial tract within the CNS. The slowly adapting neurons appear later in development and project into the lateral tract. Thus, within this set of mechanosensory neurons there exists a 3-way correlation between birth date, projection, and physiology.

\section{Electrical stimulation}

Adaptation to a mechanical stimulus may occur during the processes of coupling, transduction, and encoding. The latter 2 mechanisms involve properties intrinsic to the membrane of the sensory neuron, whereas coupling may involve biomechanical characteristics of the accessory structures of the integument. In order to specify the process responsible for adaptation in wing campaniform sensilla, we bypassed mechanical coupling by stimulating the sensory neurons directly with inward positive current. The adaptation rate within each class of sensory neurons was similar under conditions of constant mechanical and constant electrical stimulation. In the experiment shown in Figure $6 \mathrm{~A}$, a rapidly adapting neuron (ACV) responded to electrical stimulation with 1 or 2 action potentials at the onset of the current pulse, whereas a slowly adapting neuron (L3-1) contin- 
A
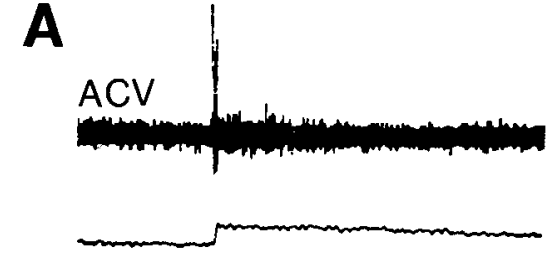

B

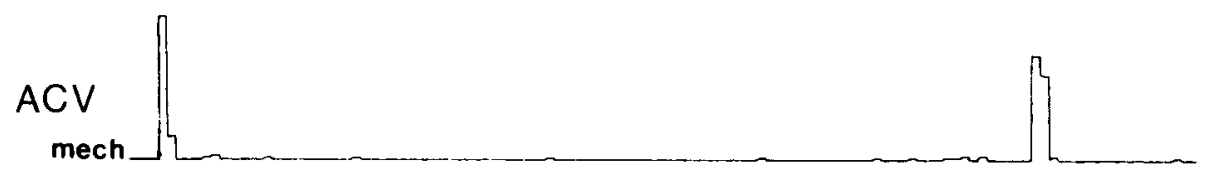

elec

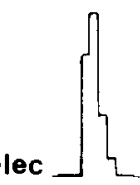
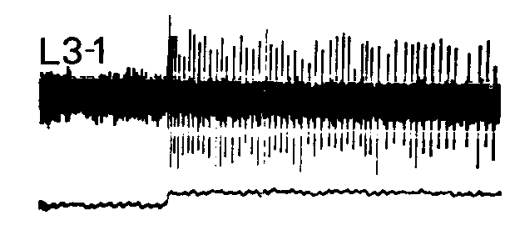

Figure 6. Comparison of electrical and mechanical stimulation of the ACV and L3-1 neurons in $D$. virilis. $A$, Extracellular recordings showing response to positive current directed inward across the cuticle of the sensillum. The ACV neuron responded to the current pulse with 2 spikes at the onset of stimulation. Similar stimulation of the L3-1 neuron resulted in a tonic discharge. Calibration bar, $50 \mu \mathrm{V}, 40 \mathrm{nA}, 200$ msec. $B$, Poststimulus time histograms of the impulse activity of neurons under conditions of mechanical and electrical stimulation. Stimuli lasted $5 \mathrm{sec}$, and responses were averaged over 16 trials. Electrical stimulation elicited responses that closely mimicked those seen under conditions of mechanical deformation. The principal exception was that electrical stimulation often did not elicit an off-response in the ACV neuron. The decreased frequency at the onset of electrical stimulation of the L3-2 neuron is an artifact due to a de shift in the baseline caused by the electrical stimulus, which temporarily lowered the spikes below the threshold of the window discriminator. Calibration bar, $50 \mathrm{~Hz}, 1$ sec.

Figure 7. Distribution of presumably homologous campaniform sensilla on flies of 4 dipteran genera. Wing size and campaniform placement is based on camera lucida drawings of 40 wings from each species, from which mean values for length, area, and position of sensilla were calculated. The wings shown in this figure were single specimens that best represented the mean values. All wings drawn to the same scale. Abbreviations: $D, D$. melanogaster; $M, M$ domestica; $C$, Calliphora sp.; $S, S$. bullata. Calibration bar, $1 \mathrm{~mm}$. 
L3-2 L3-1
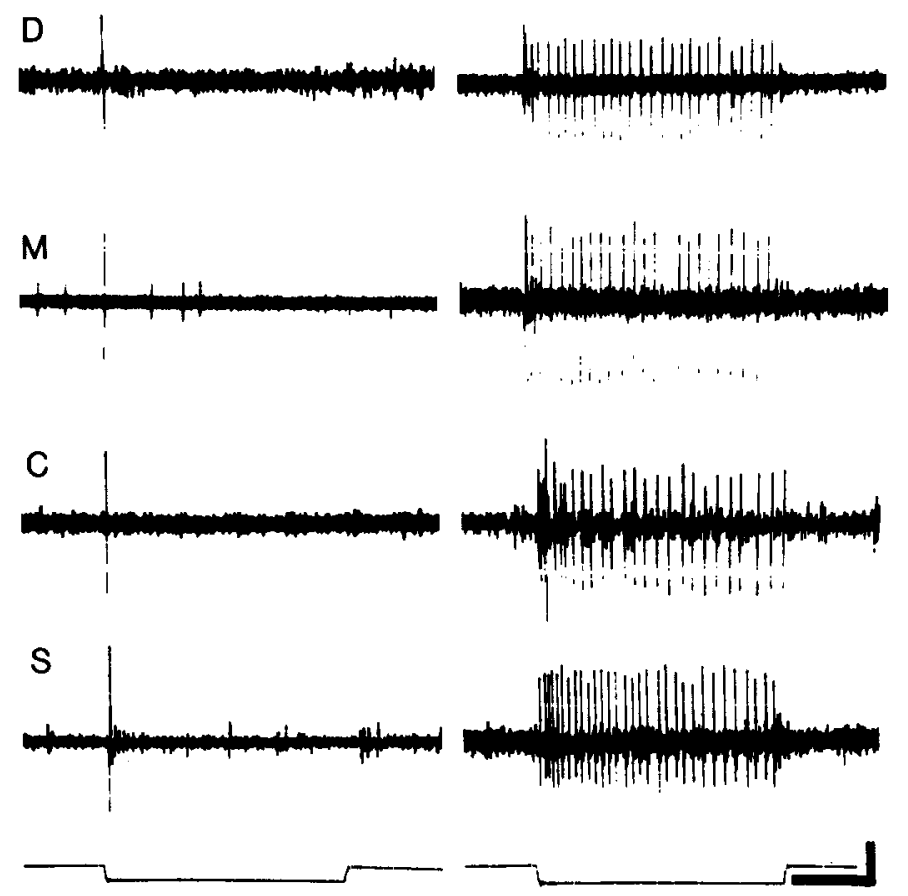

Figure 8. Extracellular recordings of responses from homologous campaniform neurons from 4 dipteran genera. Stimulus paradigm is identical to that of Figure 3, species abbreviations as in Figure 7. Recordings from the rapidly adapting L3-2 neuron are shown in the left column, recordings from the slowly adapting L3-1 neuron are shown on the right. Sensory neurons of positionally homologous campaniform sensilla have similar adaptation rates. Calibration bar, $100 \mu \mathrm{V}$ for lefi column, $50 \mu \mathrm{V}$ for right column; $200 \mathrm{msec}$ for all traces.

ucd firing for the duration of the stimulus. Poststimulus time histograms of ACV and L3-1 responses to repeated $5 \mathrm{sec}$ mechanical and electrical stimuli of the same shape are shown in Figure $6 B$. The histograms are extremely similar except for the lack of an off-response when the phasic neuron (ACV) was stimulated electrically. These results suggest that the rate of adaptation is set primarily by intrinsic electrical properties of the sensory neurons.

\section{Comparative studies}

Figure 7 shows maps of the distribution of campaniform sensilla on the wings of flies from 4 different genera: Drosophila, Musca, Calliphora, and Sarcophaga. The only notable difference in the arrangement of the sensilla is the distal and closely spaced positioning of L3-2 and L3-3 in the larger flies compared with their even spacing in Drosophila.

The response characteristics of the sensory neurons are qualitatively similar for positionally homologous sensilla, as indicated in Figure 8 . The distinction between rapidly and slowly adapting cells is maintained across the 4 genera, and even the rates of adaptation are similar. In addition, the extracellular impulses of the rapidly adapting cells were always 2-3 times larger than the impulses of the slowly adapting neurons, possibly reflecting consistent differences in axon diameter. These results suggest that within the large group muscoid flies these wing campaniform neurons comprise an evolutionarily conservative set of sensory cells.

\section{Discussion}

Correlation between physiology and central projection

We have established a strong 3-way correlation between the time of birth and differentiation, the central projections, and the intrinsic response properties of a small, defined population of sensory neurons (Fig. 9). A conventional topographically organized pattern of projections is lacking in this system (Palka et al., 1986). Nevertheless, the spatial segregation of the afferent axons within the CNS according to response properties is reminiscent of the cortical columns of slowly and rapidly adapting neurons in the mammalian somatosensory system, in which topographic maps are a prominent feature (Sur et al., 1984). Perhaps the segregation of submodalities is a general organizational principle across diverse animal taxa. The development of different classes of neurons within discrete time windows might contribute to the establishment of that segregation.

A discussion of the possible causal relationships between developmental timing and central projection has been offered by Palka et al. (1986). Here we consider several hypotheses that might account for the correlation between physiology and central projections.

1. The physiological properties of campaniform sensory neurons are determined near the time of birth, long before the axons have reached the CNS. At the same time, but by a separate process, the pathway selection mechanism of the neurons is established. For example, for strict correlation between physiology and central projection might come about if guidance molecules that control pathway choice and membrane channels that establish adaptation rate were both regulated by the same hormone. Neurons might acquire different characteristics depending upon the prevailing hormone level at the time of their differentiation. In this scenario there is no causal interaction between physiology and central projection, and both characteristics are independently correlated with the time of differentiation. If this view is correct, it might be possible to decouple these 2 properties experimentally. The properties of the L3-v neuron in normal, wild-type flies give some indication that decoupling might be possible. This neuron is the first of the late-developing group to be born and differentiate, and it also has the greatest tendency to show a phasic component in its otherwise slowly adapting response to prolonged stimulation. Its axon is the most richly branched within the lateral tract, but it shows no detectable tendency to enter the medial tract (Palka et al., 1986). Thus, it could be regarded as leaning towards intermediate physiology, but not intermediate morphology, and therefore as showing at least slight decoupling of these 2 phenotypes. This would be consistent with the view that response physiology and choice of central pathway are established independently, even though both are strongly correlated with time of development.

2. The sensory neurons, though physiologically undetermined when arriving in the CNS, are committed to a particular pathway. The differences between rapidly adapting and slowly adapting cells are induced by chemical or electrical feedback, which is different in the 2 central target regions. This hypothesis could be tested if wings could be reared to maturity while separated from the CNS, either by culturing in vitro or by implantation into host flies.

3. The elcetrical propertics of the ncurons arc cstablished at the time of differentiation, and they determine pathway choice in the CNS. This alternative is unlikely since the elimination of action potentials in bristle neurons by the nap and para mu- 


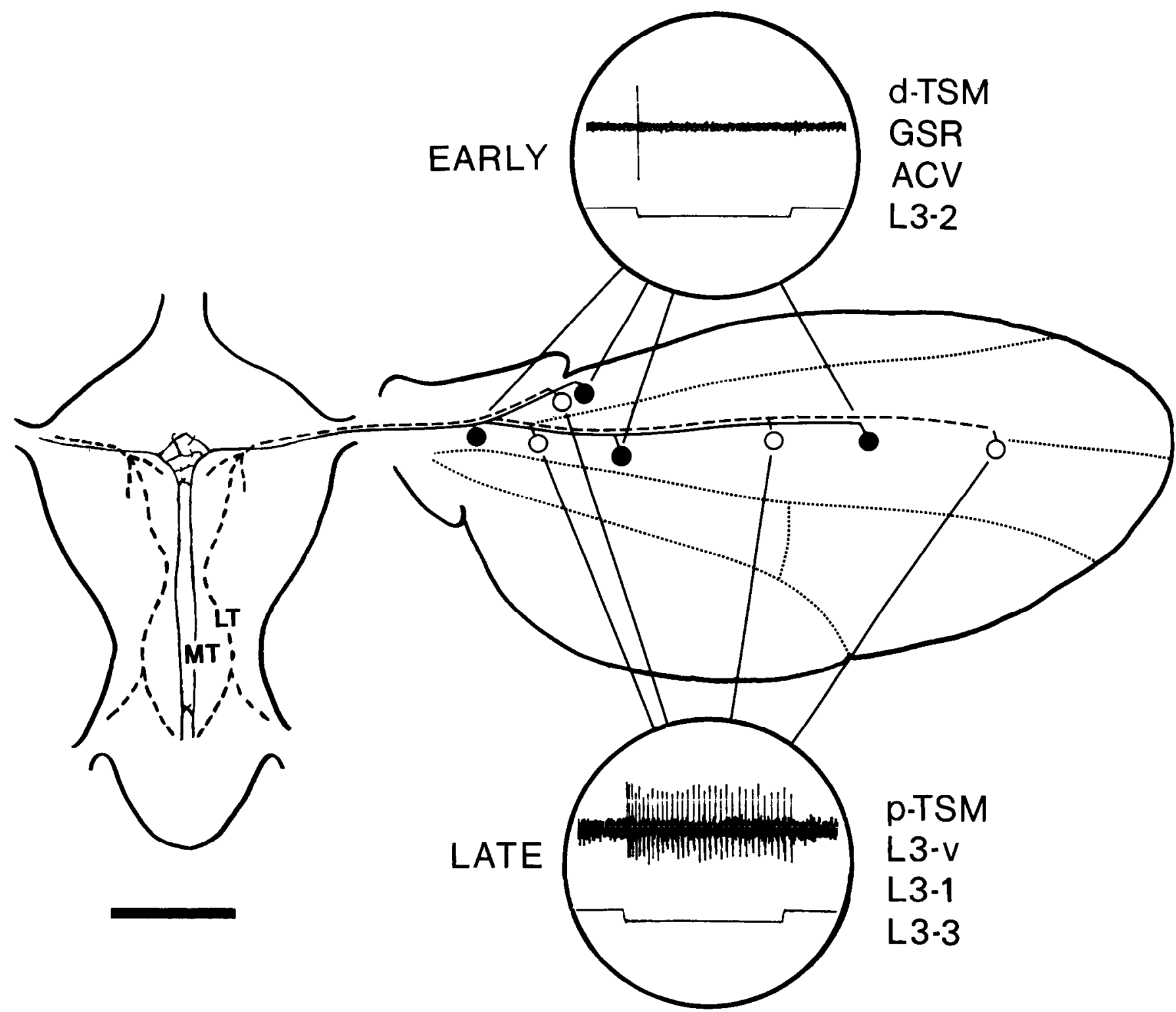

Figure 9. Diagram indicating the correlation between birthdate, central projection and physiology of the campaniform sensilla. Rapidly adapting neurons are born and differentiate in an early wave and follow the medial tract. Slowly adapting neurons arise later in development and follow the lateral tract.

tations in D. melanogaster does not affect their central projection patterns (Burg and $\mathrm{Wu}, 1986$ ). However, it is still possible that electrical activity associated with the generation of electrical responses in the dendrites might influence pathway choice.

At present, the simplest and most attractive hypothesis is that channel molecules and pathway choice molecules are expressed in a way that is independently linked to the time of differentiation.

The regulation of the time of birth and differentiation might be one mechanism that generates neuronal diversity. The hormonal environment of neurons developing in successive cohorts is different and could regulate the expression of different channel proteins and substrate recognition molecules. However, such a mechanism could not be general, for it has been shown in the CNS of grasshopper (Raper et al., 1983) and zebrafish (Mendelson and Kimmel, 1986) that the axons of neurons born at the same time can make divergent pathway choices. Nonethe- less, the time-physiology-pathway correlation we have observed is not unique, having been reported also in the cat visual system (Walsh et al., 1983; Walsh and Polley, 1985). It would seem appropriate to search for this correlation in other systems as well.

\section{Positioning of campaniform sensilla on the wing}

Although the 2 classes of campaniform neurons are not segregated into 2 discrete regions of the wing surface, their placement does suggest a distinct spatial pattern. The 6 campaniform sensilla that lie on the third vein and radius (GSR, L3-v, ACV, L31, L3-2, L3-3), and the 2 sensilla on the margin (p-TSM and d-TSM) are arranged in an alternating pattern, such that neurons of the same physiological class are never nearest neighbors (Fig. 9). The pattern is the same whether L3-2 and L3-3 are evenly spaced as in Drosophila or occur as a distal pair as in the 3 genera with larger wings (Figs. 7 and 8 ). This distribution may 
be an adaptive requirement for the function of these sensilla, and the conservation of physiological characteristics across several genera might reflect a selection pressure common throughout the Diptera. Alternatively, the alternating pattern might be a constraint imposed by phylogenetically conservative developmental mechanisms underlying the differentiation of these neurons.

The actual behavioral role(s) of specific wing campaniform sensilla in Drosophila, and the functional consequences of the segregation of their projections, are unknown at present. However, the conservative nature of the wing campaniform sensilla across a broad taxonomic sequence suggests that they play quite general roles in the behavior of flies. Wings are flexible structures and bend during many behaviors, including courtship, grooming, and flight. It is likely that these neurons provide sensory feedback during flight, since this is a behavior that produces substantial wing deformation and is common to most flies. The phasic neurons appear well suited for the detection of rapid deformations, such as those produced during each wingbeat cycle. They would be expected to remain silent during slow deformations such as probably occur during certain grooming movements. Elson (1987) has recently described campaniform neurons on locust wings that provide input to identified thoracic interneurons that might be involved in modulation of the flight rhythm. As in the flies we have studied, the population of campaniform sensory cells appears to include both rapidly and slowly adapting types. Thus, these 2 basic physiological categories are recognizable in phylogenetically distant groups of insects, a situation that invites comparative studies from functional, developmental, and evolutionary points of view.

\section{References}

Burg, M. G., and C. Wu (1986) Differentiation and central projections of peripheral sensory cells with action-potential block in Drosophila mosaics. J. Neurosci. 6: 2968-2976.

Chapman, K. M., and R. B. Duckrow (1975) Compliance and sen- sitivity of a mechanoreceptor of the insect integument. J. Comp. Physiol. 100: 251-268.

Chapman, K. M., R. B. Duckrow, and D. T. Moran (1973) Form and role of deformation in excitation of an insect mechanoreceptor. Nature 197: 699-701.

Dickinson, M. H., and J. Palka (1985) Physiological characteristics of sensory neurons of Drosophila wing correlate with central projections and developmental timing. Soc. Neurosci. Abstr. 11: 164.

Dickinson, M. H., and J. Palka (1986) Homologous sensory neurons of the dipteran wing blade. Soc. Neurosci. Abstr. 12: 136.

Elson, R. C. (1987) Integration of wing proprioceptive and descending exteroceptive sensory inputs by thoracic interneurons of the locust. J. Exp. Biol. 128: 193-217.

Levine, L. (1966) Tonicity of Acheta haemolymph. Physiol. Zool. 39: 253-258.

Mendelson, B., and C. B. Kimmel (1986) Identified verebrate neurons that differ in axonal projection develop together. Dev. Biol. 188: 309313.

Murphey, R. K. (1981) The structure and development of a somatotopic map in crickets: The cercal afferent projection. Dev. Biol. 88 . 236-246.

Murphey, R. K. (1985) The afferent projection of mesothoracic bristle hairs in the cricket, Acheta domesticus. J. Comp. Physiol. A 156: 369379.

Murray, M. A., M. Schubiger, and J. Palka (1984) Neuron differentiation and axon growth in the developing wing of Drosophila melanogaster. Dev. Biol. 104: 259-273.

Palka, J., M. A. Malone, R. L. Ellison, and D. J. Wigston (1986) Central projections of identified Drosophila sensory neurons in relation to their time of development. J. Neurosci. 6: 1822-1830.

Raper, J. A., M. Bastiani, and C. S. Goodman (1983) Pathfinding by neuronal growth cones in grasshopper embryos. I. Divergent choices made by the growth cones of sibling neurons. J. Neurosci. 3: 20-30.

Strausfeld, N. J. (1976) Mosaic organizations, layers, and visual pathways in the insect brain. In Neural Principles in Vision, F. Zettler and R. Weiler, eds., pp. 245-279, Springer-Verlag, Berlin.

Sur, M., J. T. Wall, and J. H. Kaas (1984) Modular distribution of neurons with slowly and rapidly adapting responses in area $3 \mathrm{~b}$ of somatosensory cortex in monkeys. J. Neurophysiol. 51: 724-744.

Walsh, C., and E. H. Polley (1985) The topography of ganglion cell production in the cat's retina. J. Neurosci. 5: 741-750.

Walsh, C., E. H. Polley, T. L. Hickey, and R. W. Guillery (1983) Generation of cat retinal ganglion cells in relation to central pathways. Nature 302: 611-614. 